\title{
FREE VIBRATIONAL ANALYSIS OF RECTANGULAR DUCTS WITH DIFFERENT JOINT CONDITIONS
}

\author{
Nagaraja Jade, B. Venkatesham \\ Indian Institute of Technology Hyderabad, Department of Mechanical and Aerospace Engineering, Telangana, India \\ e-mail:me12p1002@iith.ac.in; venkatesham@iith.ac.in
}

\begin{abstract}
Rectangular ducts are fabricated using thin sheets of a metal with different joint conditions. Due to the presence of these joints, ducts deviate from their assumptive ideal shapes. The objective of the present work is to study the effect of different joint conditions and wall thickness on dynamic characteristics of the ducts. Experimental Modal Analysis (EMA) is performed on three rectangular ducts of different joint types. Numerical Modal Analysis (NMA) is also performed by considering that the ducts are of ideal rectangular shape (without joint condition). Correlation analysis is performed between EMA and NMA in terms of Modal Assurance Criteria (MAC), Relative Frequency Difference (RFD) plots and auto-MAC. It is observed that natural frequencies are in good agreement but there is a discrepancy in mode shapes.
\end{abstract}

Keywords: rectangular duct, duct joint, experimental modal analysis, modal assurance criteria

\section{Introduction}

Dynamic characteristics such as natural frequencies, mode shapes and damping factors of an engineering structure are determined by modal analysis, either analytically, numerically or experimentally (Ewins, 2000a). Finite Element Analysis (FEA) is a quick and easy way to know dynamic characteristics of any structure, but determination of its damping parameters is difficult. Another method is using the analytical technique for solving mathematical models which represent the actual structure. Experimental Modal Analysis (EMA) is most common and effective method to determine, improve and optimize dynamic characteristics of an engineering structure (Ewins, 2000a). The analytical and FEA models can be verified using EMA. Kennedy and Pancu (1947) first proposed the most significant method of EMA before Fast Fourier Transforms (FFT) was invented. Fu and He (2001) provided a detailed theory of analytical and experimental modal analysis to characterize the dynamic behavior of any structure. Significant advances in experimental techniques enhanced the modeling of a structure and determining its dynamic properties. Experimental results can be used as reference (i) to modify the existing structure and to optimize the structure design by an iteration process, (ii) validate analytical models in order to refine future designs (Maia, 1988).

Prior to the actual modal test, it is essential to identify the optimal number, locations of excitation and the response measurement points to acquire all the modes excited within the interested frequency range. In the literature, few researchers proposed techniques for calculating the optimum number and position of measurements for the modal test based on Frequency Response Functions (FRF) (Schwarz et al., 2002) and finite element analysis (Linehan and Napolitano, 2012). Similarly, it is important to choose an apt excitation method, as the influence of each mode on the overall response of the structure depends on the type of the excitation. Shaker and impact hammer are the most common types of excitations. Dynamic characteristics are extracted from the measured FRF's using curve fitting methods. Selection of the curve fitting techniques 
is important in extracting dynamic characteristics from the measured time domain or frequency domain data (Maia, 1988; Ibrahim, 1999). In this regard, different algorithms are developed, which are based on Single-Degree of Freedom (SDOF) and Multi-Degree of Freedom (MDOF) methods (Brandon and Cowley, 1983; Maia and Silva, 1997). The modal vectors obtained from experimental measurements are compared using numerical modal vectors by correlation analysis, in terms of the Modal Assurance Criteria (MAC) plot. Allemang (1999) and Ewins (2000b) discussed various existing methods to perform systematic comparison and correlation between two sets of vibration data, namely numerical and experimental data.

Different analytical methods are proposed in the literature for calculating natural frequencies and mode shapes of rectangular and circular ducts with different axial boundary conditions (Blevins, 1979; Lee, 1993; Chavan and Venkatesham, 2015). Majorly in the literature, an ideal rectangular duct geometry is considered in analytical and numerical studies. However, practical ducts are fabricated with different joint conditions. Hence, it is important to study the effect of these joints on dynamic characteristics. Xiao et al. (2011) studied the effect of variation in geometry, boundary conditions and material properties on the vibration characteristics of the cylinder. Zaman et al. (2013) discussed the effect of bolted joints on dynamic characteristics of the beam and frame structures. They showed that natural frequencies are varied due to the presence of joints. Adequate literature is unavailable to show the joint effect and geometric distortion on the dynamic characteristics.

The current paper discusses the effect of joint conditions (used for fabrication) and wall thickness on dynamic characteristics of a duct, experimentally. To understand the effect of these parameters, the present study considers three different ducts which differ mainly by the type of joints (such as Pittsburgh lock joint, welded joint and adhesive joint) used in fabrication. Ducts with different wall thickness are considered to study the modal density effect. In the present paper, Section 2 describes theoretical background for estimating the dynamic characteristics and correlation analysis. Pre-test analysis, the experimental test setup, modal test procedure and numerical modal analysis are explained in Section 3. In Section 4, experimental and numerical modal analysis results are discussed while in Section 5 conclusions are presented.

\section{Theoretical background}

This Section provides a brief theoretical explanation about the estimation of dynamic characteristics such as natural frequencies and mode shapes of a structure. It also outlines the available techniques to perform the correlation of experimental and numerical results after modal analysis. Here, dynamic characteristics can also be referred as modal parameters.

\subsection{Natural frequencies and mode shapes}

The conventional hypothesis for calculating dynamic characteristics of a structure is using the equation of motion. For free vibration analysis of a multi-degree freedom system, this is written in terms of mass, stiffness and damping matrices as follows (Blevins, 1979)

$$
\mathbf{M}_{N \times N} \ddot{\mathbf{x}}_{N \times 1}+\mathbf{C}_{N \times N} \dot{\mathbf{x}}_{N \times 1}+\mathbf{K}_{N \times N} \mathbf{x}_{N \times 1}=\mathbf{0}
$$

where $N$ is the number of degrees of freedom (DOF) in the system, $\mathbf{M}, \mathbf{C}$ and $\mathbf{K}$ designate the matrices of mass, damping, and stiffness respectively. $\ddot{\mathbf{x}}, \dot{\mathbf{x}}$ and $\mathbf{x}$ are the second, first and zeroth derivatives of system displacements. Solving Eq. (2.1), which is similar to the general eigenvalue problem, results in $N$ eigenvalues (natural frequencies) and $N$ eigenvectors (mode shapes)

$$
\lambda_{i}=-\xi_{i} \omega_{i}+\mathrm{j} \omega_{i} \sqrt{1-\xi_{i}^{2}}
$$


where $\lambda_{i}$ is the $i$-th pole of the FRF, $\omega_{i}$ is the $i$-th circular natural frequency of the system and $\xi_{i}$ is the damping ratio for the $i$-th natural frequency of the system.

\subsubsection{Numerical modal analysis (NMA) approach}

Equation (2.1) can be solved using an analytical and FEA method. Calculation of the mass and stiffness matrix for simple geometry is straightforward, but for complex geometries, FEA is an appropriate method. In this method, continuous geometry is discretized with the finite number of elements. So, it is an approximation to infinite degrees of freedom system with finite degrees. This assumption leads to discretized equations which can be solved using different numerical methods for eigenvalues and eigenvectors. For this numerical analysis, convergence study is required for accurate result predictions. This methodology is well established in the literature and a good number of commercial softwares are available in the market. The prediction analysis can be referred to as numerical modal analysis in this paper.

\subsubsection{Experimental modal analysis (EMA) approach}

In the EMA approach, dynamic characteristics of a structure are estimated using measured frequency response functions (FRF). These FRFs can be obtained by exciting the structure and measuring the system inputs and outputs at various positions on the structure. There are mainly three important steps involved for performing EMA, namely, establishing the measurement set-up, acquiring the data and extracting the modal parameters. The detailed procedure for the experimental test setup and extraction of natural frequencies and mode shapes is given in Section 3.

\subsection{Modal assurance criteria}

There are different methods available for performing the correlation analysis of EMA and NMA results (FEA analysis). The frequently used method is the modal assurance criterion (MAC) plot. It compares the EMA vector and FEA vector. MAC is a scalar quantity and it varies between 0 and 1 . If two vectors are the same, then MAC value is 1 . Generally, MAC value of 0.6 and above is considered as acceptable (Vacher et al., 2010).

If $\boldsymbol{\Phi}_{i}^{E M A}$ is the modal vector from EMA and $\boldsymbol{\Phi}_{j}^{F E M}$ is from FEA, then MAC is defined as

$$
M A C_{i j}=\frac{\left|\boldsymbol{\Phi}_{i}^{E M A} \boldsymbol{\Phi}_{j}^{F E M}\right|^{2}}{\left(\boldsymbol{\Phi}_{i}^{E M A} \boldsymbol{\Phi}_{i}^{E M A}\right)\left(\boldsymbol{\Phi}_{j}^{F E M} \boldsymbol{\Phi}_{j}^{F E M}\right)}
$$

where $\boldsymbol{\Phi}$ is the modal vector, $i$ and $j$ are the mode numbers of the experimental and numerical results, respectively.

\subsection{Relative frequency difference}

Relative Frequency Difference (RFD) is a simple plot which calculates the RFD between two different natural frequency sets. RFD values should be as low as possible, typically, an error of $10 \%$ is acceptable. Higher values are observed while moving away from the diagonal elements (Ewins, 2000b). The acceptable error may vary for different types of test structures and testing methods.

If $f_{e}^{i}$ is the experimental natural frequency of $i$-th mode and $f_{n}^{j}$ is the numerical natural frequency of $j$-th mode, then RFD is given as

$$
R F D_{i j}=\frac{f_{n}^{j}-f_{e}^{i}}{f_{e}^{i}}
$$




\subsection{Drive point residue}

Drive Point Residue (DPR) can be specified as equivalent to modal participation factors, and DPR measures how well each mode is excited or its participation in the overall response of the structure at a chosen driving point. The definition of DPR for mode $k$ and mode $i$ is given by Allemang (1999)

$$
D P R_{k}(i)=\frac{\Phi_{i k}^{2}}{2 m_{k} \omega_{k}}
$$

where $\boldsymbol{\Phi}$ is the mode shape (modal vector), $\omega$ is the circular natural frequency, $m$ is the modal mass. The degrees of freedom (DOF) with the maximum average DPR for chosen mode shapes are considered to be a better excitation DOFs for the interesting set of target modes.

\section{Experimental setup and numerical analysis}

The present Section discusses the pre-test analysis, setup and procedure for experimental and numerical modal analysis. Firstly, specifications such as dimensions, type of the joint for the fabrication and material properties of the different ducts are discussed. Three different types of rectangular ducts are considered in the analysis as shown in Fig. 1 are as follows:

Case 1. Duct with Pittsburgh lock joint (Duct 1)

In this configuration, the rectangular sheet of mild steel is bent at three corners, and the edges are joined using Pittsburgh locking mechanism. Dimensions of the duct is: $0.3 \mathrm{~m} \times 0.4 \mathrm{~m} \times 1.2 \mathrm{~m}$ with 20 gauge $(\sim 1 \mathrm{~mm})$. Material properties are: Young's modulus $E$ is $200 \mathrm{GPa}$, Poisson's ratio $\mu$ is 0.3 and density is $7850 \mathrm{~kg} / \mathrm{m}^{3}$.

Case 2. Duct with welded joints at four edges (Duct 2)

The duct is fabricated by welding joints at four right angle corners of individual plates. The arc welding technique is used for fabrication. Two pairs of plates with dimensions $0.3 \mathrm{~m} \times 1.5 \mathrm{~m}$ and $0.4 \mathrm{~m} \times 1.5 \mathrm{~m}$ are used. The overall dimensions of the duct are: $0.3 \mathrm{~m} \times 0.4 \mathrm{~m} \times 1.5 \mathrm{~m}$ with 10 gauge $(\sim 3 \mathrm{~mm})$. Duct material properties are: Young's modulus $E$ is $200 \mathrm{GPa}$, Poisson's ratio $\mu$ is 0.3 and density is $7850 \mathrm{~kg} / \mathrm{m}^{3}$.

Case 3. Duct with adhesive joint (Duct 3)

In this configuration, to fabricate a duct closer to an ideal rectangular shape, an acrylic sheet with the smooth plane surface is used. The individual plates are joined by an adhesive joint. Cyanoacrylate adhesive is used which establishes strong bond and smooth connectivity between the plates. The overall dimensions of the duct are: $0.206 \mathrm{~m} \times 0.258 \mathrm{~m} \times 0.9 \mathrm{~m}$ with 4 gauge $(\sim 5 \mathrm{~mm})$. The material properties are: Young's modulus $E$ is $3.2 \mathrm{GPa}$. Poisson's ratio $\mu$ is 0.4 , and density is $1100 \mathrm{~kg} / \mathrm{m}^{3}$.

\subsection{Numerical modal analysis}

Numerical modal analysis is performed on all duct cases using commercial software (ANSYS) to calculate natural frequencies and mode shapes of the ducts. However, the description is provided for Case 1 only since a similar procedure applies to all cases. Rectangular duct of dimensions $0.3 \mathrm{~m} \times 0.4 \mathrm{~m} \times 1.2 \mathrm{~m}$ with wall thickness of $1 \mathrm{~mm}$ is modelled (as ideal duct i.e. without joint condition) and meshed using SHELL 63 elements. An element size of $0.005 \mathrm{~m}$ is chosen for meshing based on convergence study. The simply supported boundary condition is applied at 


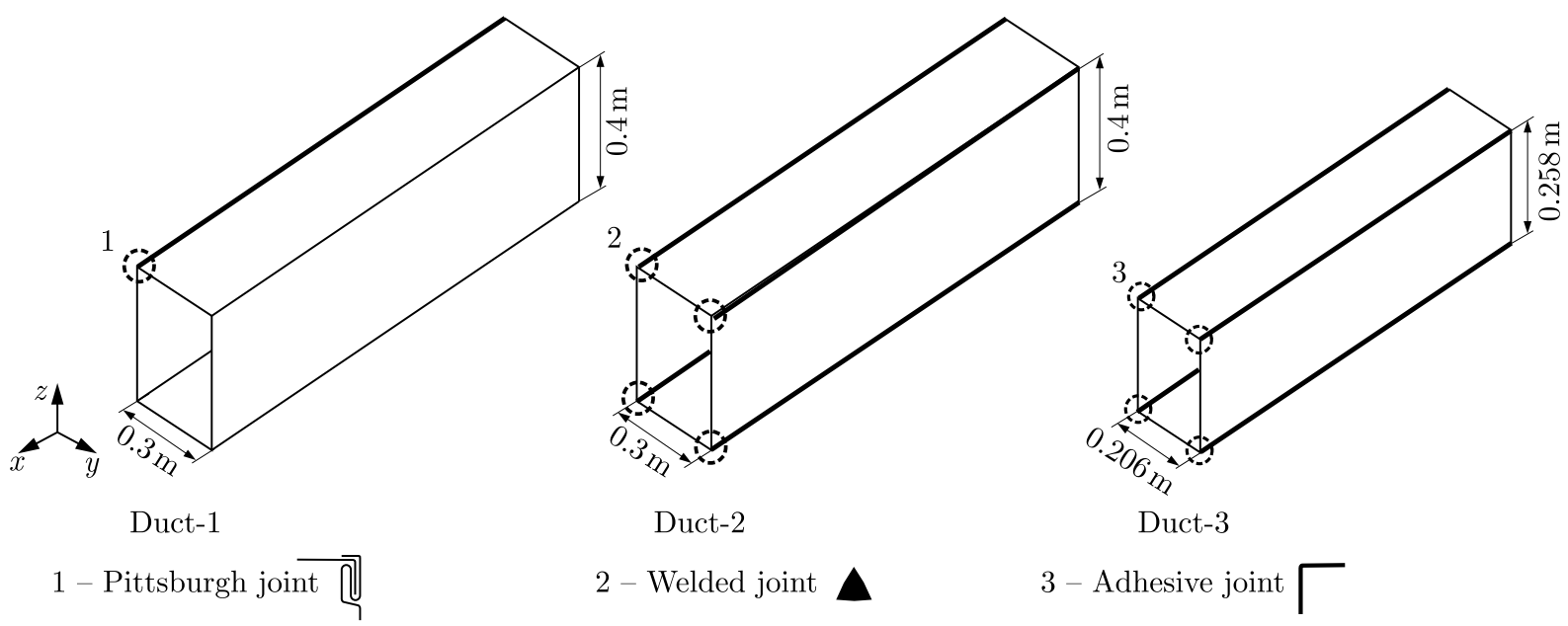

Fig. 1. Duct configurations with different joint conditions used in the experimental modal analysis

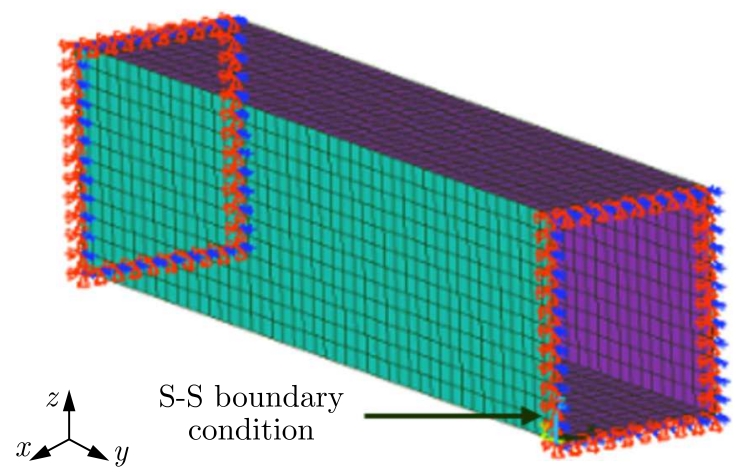

Fig. 2. Finite Element Model of a rectangular duct with simply supported boundary conditions

both ends of the duct. Figure 2 shows the meshed model with boundary conditions of the duct structure.

Mild steel properties such as: Young's modulus $200 \mathrm{GPa}$, density $7850 \mathrm{~kg} / \mathrm{m}^{3}$, Poisson's ratio 0.3 and structural damping ratio of 0.01 are applied to the duct structure. Numerical modal analysis is performed to calculate the modal parameters up to $200 \mathrm{~Hz}$ frequencies. These parameters are used for pre-test analysis and also for correlation with EMA results.

\subsection{Pre-test analysis}

Preceding to the EMA, a pre-test is performed to find an appropriate reference point and response locations to mount the accelerometers. Thus, accurate structural modal parameters can be captured from modal test. This analysis is done for all cases within the interested frequency range of 10-200 Hz. The results are given for Case 1 only, as shown in Fig. 3, which indicates the location, number of excitations and response measurement points. Figure 3a shows that nodal point numbers, 128 with $+x$-direction and 84 with $+z$-direction are suitable DOFs for excitation in the experimental modal test. The locations of these points are $(x=0.3, y=0.1, z=0.24)$ and $(x=0.18, y=0.5, z=0.4)$ as shown in Fig. 3b. Here, due to closely spaced modes, 160 response measurement points are considered to efficiently characterize individual mode shapes as shown in Fig. 3c. 
(a)

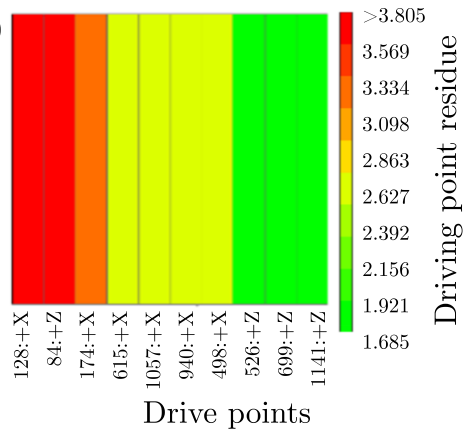

(b)

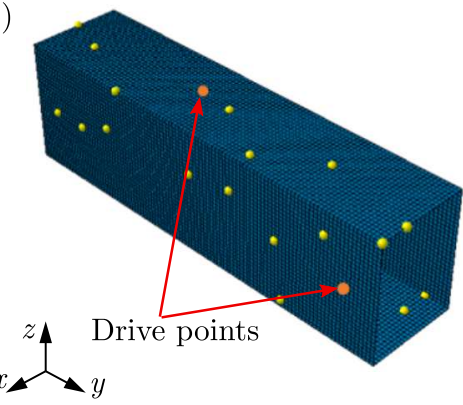

(c)

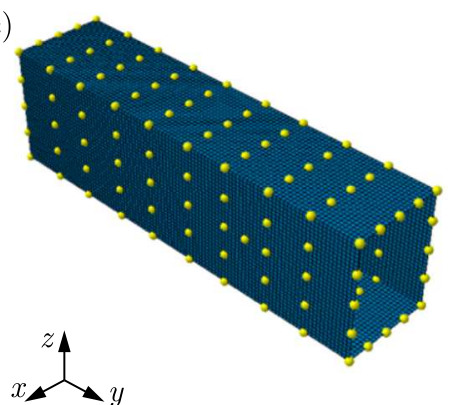

Fig. 3. Pre-test analysis: (a) drive point residue, (b) drive points to excite the structure, (c) response measurement points highlighted with dots

\subsection{Experimental test setup}

Preparation of measurement set-up involves preparation of boundary conditions, selection of the excitation method, type of transducers and measurement locations. EMA is performed on ducts in Case 1 and Case 2 with simply supported (S-S) boundary condition (BC) and Case 3 with free-free (F-F) BC as shown in Fig. 4. The S-S condition is achieved by making the contact point with duct walls at the front and rear portion of the duct. Steel balls are inserted into the rectangular frames to make them as a point of contact with the duct at the front and rear cross sections. The F-F boundary condition is achieved by suspending the duct with flexible bungee ropes.

(a)

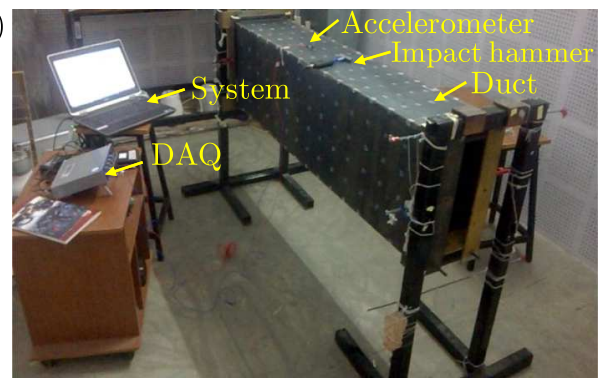

(b)

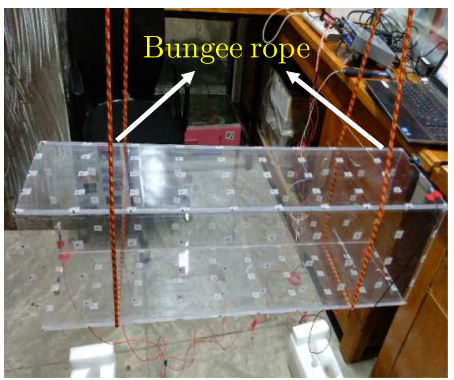

Fig. 4. EMA test setup: (a) simply supported boundary conditions, (b) free-free boundary condition

The modal test is performed by the roving hammer method. As the reciprocity principle holds good, the drive points obtained from pre-test analysis are used as response points. High sensitivity, lightweight tri-axial and uni-axial accelerometers of IEPE type are used to capture the vibration response. Weight of tri- and uni-axial sensors are 3 and 10 grams, respectively. An impact hammer of IEPE type with 100 gram head weight is used for excitation in order to induce vibrations in the structure. Once the measurement set-up is established, FRFs are measured with appropriate settings such as sampling frequency, bandwidth, number of samples and windowing function. It is important to verify the quality of acquiring data to ensure consistent measurements by observing the input excitation, coherence and FRFs. The measured FRFs are used to extract modal parameters of the duct. There are several methods to extract modal parameters in both the time domain and frequency domain as mentioned in Section 1. Multivariate Mode Indicator Function (MvMIF) is used for extracting modal parameters. Selection of poles is verified by comparing the measured drive point FRF and synthesized MvMIF curve. Figure 5 shows the stability diagram for multi-degree of freedom (MDOF) analysis to select poles and vectors. Selected stabilized poles are indicated by solid squares. 


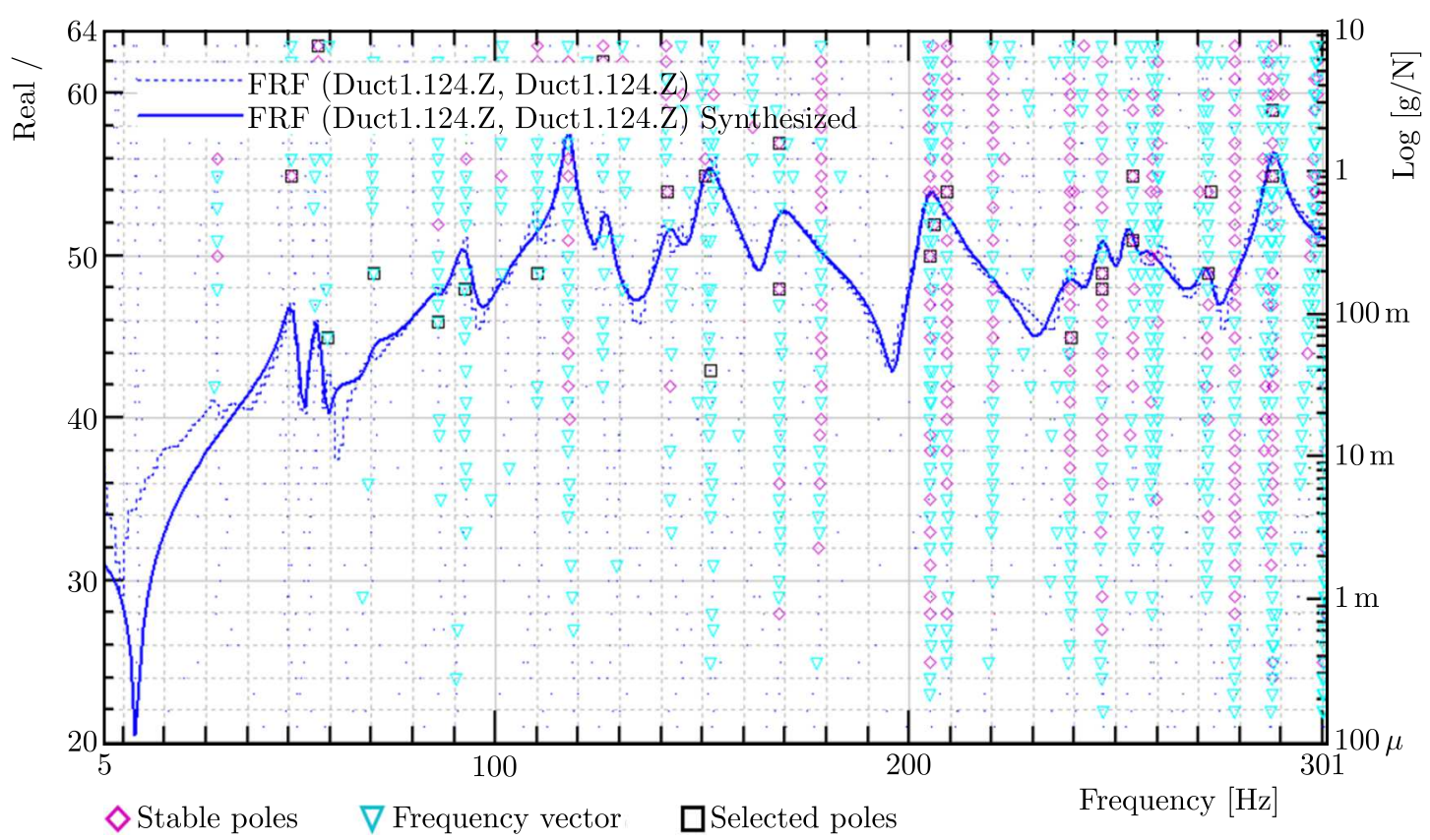

Fig. 5. Stability diagram for MDOF analysis of the duct as a function of frequency

\section{Results and discussion}

Experimental and numerical modal analysis results corresponding to all three ducts cases are discussed here. As a primary step, auto-MAC analysis is performed. Natural frequencies obtained from the experimental and numerical analysis are compared in the form of tables and RFD plots. Next, mode shapes are compared by visual inspection and correlation analysis in terms of MAC values.

\subsection{Auto-MAC correlation}

Auto-correlation analysis is performed for experimental mode shapes to (i) verify that a sufficient number and location of measurements are chosen for performing the model test and (ii) determine the correlation of individual modes with other modes within the interested frequency range (Linehan and Napolitano, 2012). Auto-MAC plots are shown in Fig. 6 for three cases (Ducts 1-3). The auto-MAC matrix is almost diagonal because the individual modal vector is orthogonal to other modal vectors.

(a)

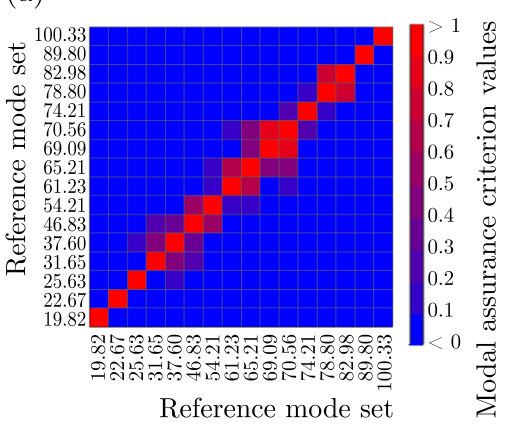

(b)

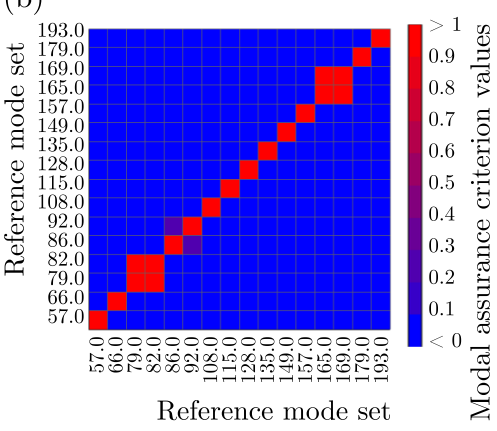

(c)

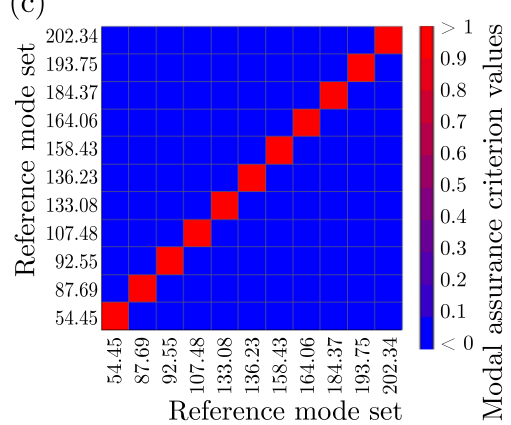

Fig. 6. Auto-MAC plots of experimental modal analysis results for all three ducts cases

From the auto-MAC plot of Case 1, it is observed that some individual modes are correlated with multiple modes. This is due to close spacing of modes, which leads to poor MAC values. 
For example, mode 4 is correlated with modes 5, 6 and mode 10 with modes 9,11 . In Case 2, most of the off-diagonal MAC values are very low except for modes 3, 4 and modes 14, 15. This is due to symmetric modes and existence of welded joints. Auto-MAC plot in Case 3 is perfectly diagonal.

\subsection{Comparison of natural frequencies}

Analytical natural frequencies for Duct 1 and Duct 2 are calculated using the analytical models available in literature (Chavan et al., 2015). Comparison of experimental, numerical and analytical natural frequencies for Duct 1 and Duct 2 is shown in Table 1. It is observed that analytical and numerical results are in good agreement and match well with experimental results with less than a $10 \%$ error. However, in Duct 1, modes 5, 8 and 10 are not captured in EMA due to higher modal density and the symmetric condition of duct geometry (as the repeated modes are difficult to be obtained from an experiment). Similarly, mode 9 in Duct 2 case.

Table 1. Comparison of natural frequencies for Duct 1 and Duct 2

\begin{tabular}{|c|c|c|c|c|c|c|}
\hline \multirow{2}{*}{$\begin{array}{c}\text { Mode } \\
\text { No. }\end{array}$} & \multicolumn{3}{|c|}{$\begin{array}{c}\text { Duct 1 } \\
\text { Natural frequencies [Hz] }\end{array}$} & \multicolumn{3}{c|}{ Dutural 2 } \\
& Exequencies [Hz] \\
\hline \hline 1 & 19.82 & 20.64 & 20.69 & 57.00 & 58.71 & 58.79 \\
\hline 2 & 22.67 & 25.52 & 25.58 & 66.00 & 67.77 & 67.91 \\
\hline 3 & 25.63 & 26.85 & 27.07 & 79.00 & 76.96 & 77.87 \\
\hline 4 & 31.65 & 30.70 & 30.91 & 82.00 & 83.05 & 83.27 \\
\hline 5 & - & 33.77 & 33.85 & 86.00 & 84.12 & 84.84 \\
\hline 6 & 37.6 & 37.75 & 37.97 & 92.00 & 96.66 & 97.40 \\
\hline 7 & 41.12 & 40.03 & 40.24 & 108.00 & 104.66 & 105.00 \\
\hline 8 & - & 44.53 & 44.74 & 115.00 & 115.08 & 116.10 \\
\hline 9 & 46.83 & 45.44 & 45.54 & - & 115.53 & 116.37 \\
\hline 10 & - & 48.39 & 48.62 & 128.00 & 123.71 & 124.45 \\
\hline
\end{tabular}

Table 2 shows a comparison of natural frequencies obtained from the experimental modal test and predicted by numerical analysis for Duct 3. It shows that the predicted frequencies are well captured by the experimental modal test with less than a $5 \%$ error.

Table 2. Comparison of natural frequencies for Duct 3

\begin{tabular}{|c|c|c|c|c|c|}
\hline \multirow{2}{*}{$\begin{array}{c}\text { Mode } \\
\text { No. }\end{array}$} & \multicolumn{2}{|c|}{ Natural frequencies $[\mathrm{Hz}]$} & \multirow{2}{*}{$\begin{array}{l}\text { Mode } \\
\text { No. }\end{array}$} & \multicolumn{2}{|c|}{ Natural frequencies $[\mathrm{Hz}]$} \\
\hline & Experimental & Numerical & & Experimental & Numerical \\
\hline 1 & $\overline{54.45}$ & $\overline{54.57}$ & 6 & 133.08 & 136.46 \\
\hline 2 & - & 54.81 & 7 & 136.23 & 136.47 \\
\hline 3 & 87.69 & 89.47 & 8 & 158.43 & 160.02 \\
\hline 4 & 92.55 & 93.60 & 9 & 159.37 & 160.79 \\
\hline 5 & 107.48 & 109.81 & 10 & 164.06 & 163.01 \\
\hline
\end{tabular}

Numerical modal analysis is performed for Duct 1 with different thickness and compared with the experimental results as shown in Table 3. It is observed that the experimental results are close to the numerical results of duct with $1 \mathrm{~mm}$ thickness. It can be noticed (from Table 3 ) that as thickness of the duct wall increases (from $1 \mathrm{~mm}$ to $3 \mathrm{~mm}$ ), the natural frequency also increases. It indicates that the modes are well separated. The similar behaviour is observed between Duct 1 and 2 . 
Table 3. Comparison of natural frequencies of experimental and numerical analysis with different thickness for Duct 1

\begin{tabular}{|c|c|c|c|c|c|c|}
\hline \multirow{2}{*}{$\begin{array}{c}\text { Mode } \\
\text { No. }\end{array}$} & \multicolumn{7}{|c|}{ Natural frequencies [Hz] } \\
\cline { 2 - 7 } & Experimental & \multicolumn{5}{|c|}{ Numerical } \\
\cline { 2 - 7 } & $1 \mathrm{~mm}$ & $1 \mathrm{~mm}$ & $1.5 \mathrm{~mm}$ & $2 \mathrm{~mm}$ & $2.5 \mathrm{~mm}$ & $3 \mathrm{~mm}$ \\
\hline \hline 1 & 19.82 & 20.64 & 30.97 & 41.29 & 51.61 & 61.93 \\
\hline 2 & 22.67 & 25.52 & 38.29 & 51.05 & 63.81 & 76.57 \\
\hline 3 & 25.63 & 26.85 & 40.25 & 53.61 & 66.94 & 80.21 \\
\hline 4 & 31.65 & 30.70 & 46.04 & 61.37 & 76.70 & 92.02 \\
\hline 5 & - & 33.77 & 50.65 & 67.54 & 84.42 & 101.30 \\
\hline 6 & 37.6 & 37.75 & 56.62 & 75.49 & 94.35 & 113.20 \\
\hline 7 & 41.12 & 40.03 & 60.01 & 79.94 & 99.80 & 119.58 \\
\hline 8 & - & 44.53 & 66.78 & 89.03 & 111.26 & 133.48 \\
\hline 9 & 46.83 & 45.44 & 68.15 & 90.87 & 113.58 & 136.29 \\
\hline 10 & - & 48.39 & 72.59 & 96.78 & 120.96 & 145.14 \\
\hline
\end{tabular}

RFD plots can also be used to check the relation between measured and predicted natural frequencies. Minimum RFD value indicates that two-mode sets are close to each other. The RFD plot for all cases is shown in Fig. 7. It is observed that RFD values are minimum along the diagonal, and it is clear that both measured and predicted frequencies are in good agreement. Thus it is interpreted that material properties used in numerical simulations are appropriate (Ewins, 2000b). The RFD of experimental and numerical results is less than $10 \%$ for all the cases. It is clear from the RFD plot that experimental and numerical natural frequencies are matched well.

(a)

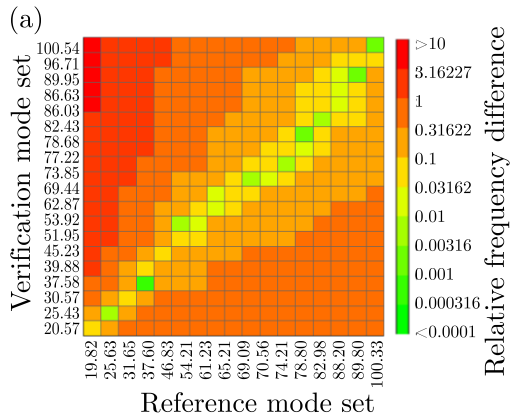

(b)

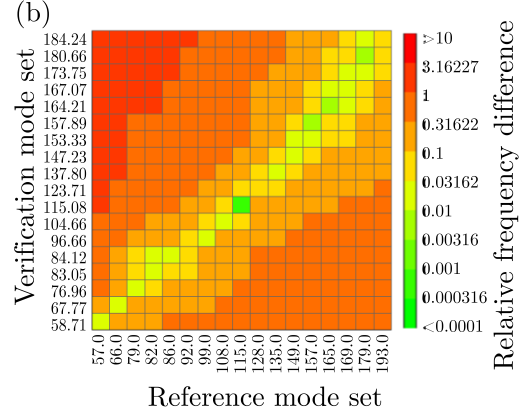

(c)

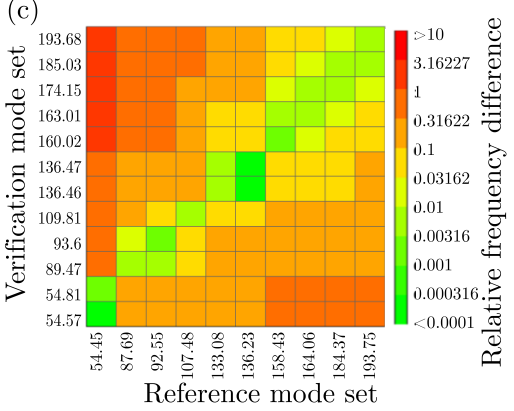

Fig. 7. Relative frequency difference plot between experimental and numerical modal analysis results for all ducts

\subsection{Mode shape comparison}

One of the quick ways to compare experimental and numerical results is the visual inspection of mode shapes. For easiness of visual observation of mode shapes, the colour bar is maintained the same in both experimental and numerical results. Figure 8 shows comparison of numerically predicted and experimentally measured mode shapes for the first four modes of Duct 1 and Duct 2. In the case of Duct 1, it is observed that there is a poor agreement between experimental and numerical mode shapes. For example, the modal displacement is not significant on the side plate for modes 1, 2 and 4 due to the joint between the top and side plate. Modal strain energy is not transferring from one plate to another due to high stiffness and irregularity of the joint. In Duct 2 case, it is observed that experimental mode shapes reasonably resemble the numerical mode shapes. 


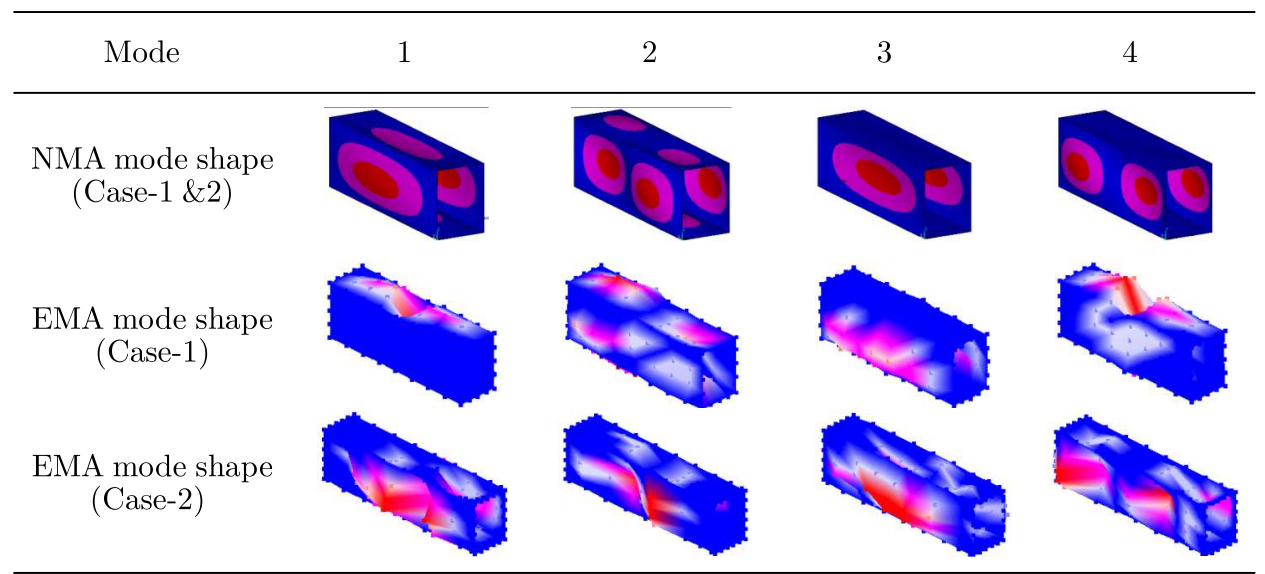

Fig. 8. Comparison of the first four mode shapes for Duct 1 (Pittsburgh lock joint) and Duct 2 (welded joints)

Figure 9 shows a comparison of the measured ang predicted mode shapes for Duct 3 . It is observed that experimental mode shapes are matched very well with numerical mode shapes except for the first mode, since it is difficult to capture twisted modes in EMA. The extent of correlations between these mode pairs can be verified by MAC values which are shown in the next Section.

\begin{tabular}{|c|c|c|c|c|}
\hline Mode & 1 & 3 & 4 & 5 \\
\hline $\begin{array}{l}\text { NMA mode } \\
\text { shape }\end{array}$ & & & & \\
\hline $\begin{array}{l}\text { EMA mode } \\
\text { shape }\end{array}$ & & & & \\
\hline
\end{tabular}

Fig. 9. Comparison of experimental and numerical mode shapes for Duct 3 (adhesive joint)

\subsection{Modal assurance criteria}

Correlated modes are calculated in terms of MAC values, which vary from 0 to 1 . Generally, the reasonable MAC value 0.6 is acceptable, but for good correlation of experimental and numerical results, MAC values should be greater than 0.9 (Vacher et al., 2010). The MAC plot for all the cases is shown in Fig. 10. In Duct 1, it is observed that only two mode pairs have MAC values greater than or equal to 0.6 and the remaining modes have a poor correlation. It clearly shows that the first numerical mode shape is correlated with the second experimental mode (MAC is 0.59). One of the higher mode pairs such as FE mode 13 and experimental mode 11 have MAC values greater than 0.8 .

The MAC plot for sixteen experimental modes and twenty-one FEA modes are calculated and plotted for Duct 2 (Fig. 10b). Few off-diagonal mode pairs display that MAC values are greater than 0.5 which indicates that one mode has a correlation with multiple modes. This is due to the presence of welded joints, which makes the structure stiffer at the joints and each wall of the duct behaves as an individual plate constrained at the edges. Experimental modes 2-4, 8 and 10 have MAC values greater than 0.6. 
The effect of thickness is also observed between Case 1 and Case 2. It shows that modal density is high in Case 1 when compared to Case 2.
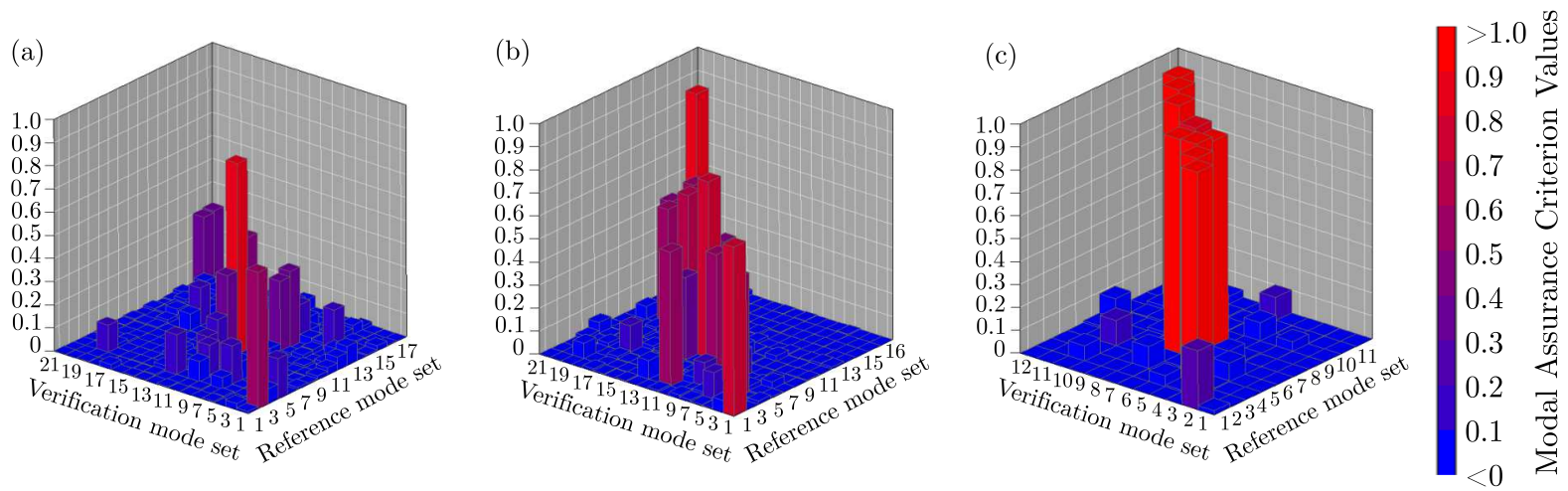

Fig. 10. Comparison of mode shapes for EMA and NMA results in terms of the Modal Assurance Criteria plot

In Duct 3, correlation analysis is done for eleven experimental modes with twelve FEA modes. From the MAC plot shown in Fig. 10c, it is observed a good agreement between the predicted and measured mode shapes except for two fundamental modes (as these are two rotated modes). However, there exists a cross-correlation between modes 7 and 8 (as they are symmetric modes) which can be corrected by updating the FEA modal model. A mode pair table for correlated mode pairs with MAC values greater than or equal to 0.9 is shown in Table 4 . It is observed that modes 3, 4 and 5 have excellent correlation with the MAC value equal to 0.99 . The results of Duct 3 (acrylic), which is close to an ideal rectangular shape without any joint problem show good correlation with MAC value of 0.99 .

Table 4. Mode pair table with MAC values for Duct 3

\begin{tabular}{|c|c|c|}
\hline $\begin{array}{c}\text { Experimental } \\
\text { natural } \\
\text { frequencies }[\mathrm{Hz}]\end{array}$ & $\begin{array}{c}\text { Numerical } \\
\text { natural } \\
\text { frequencies [Hz] }\end{array}$ & MAC Value \\
\hline \hline 87.7 & 89.5 & 0.981 \\
\hline 92.5 & 93.6 & 0.985 \\
\hline 107.5 & 109.8 & 0.992 \\
\hline 133.1 & 136.5 & 0.935 \\
\hline 158.4 & 160.0 & 0.894 \\
\hline 169.3 & 174.2 & 0.914 \\
\hline 179.8 & 185.0 & 0.928 \\
\hline 190.1 & 193.7 & 0.922 \\
\hline 87.7 & 89.5 & 0.981 \\
\hline 92.5 & 93.6 & 0.985 \\
\hline 107.5 & 109.8 & 0.992 \\
\hline
\end{tabular}

As the rectangular ducts are axisymmetric in structure, repeated roots (symmetrical modes) exist. It is difficult to capture these symmetrical modes in the experimental modal test. This can be observed from natural frequency comparison tables (for ducts of Case 1-3), where few modes are missing (Tables 1 and 2). 


\subsection{Effect of boundary condition}

To study the effect of the boundary condition on mode shapes of a duct with the joint condition, EMA is performed on Duct 2 with free-free BC along with simply supported BC. Experimental and numerical natural frequencies are in a good agreement with less than a $10 \%$ error. The experimental mode shapes are compared with the numerical mode shapes, and the corresponding MAC plot is shown in Fig. 11. It can be observed that a single mode is correlated with multiple modes, and MAC vales are low. Similar behaviour is also observed in the MAC plot of Duct 2 with S-S BC (Fig. 10b). So, it is clearly observed that the presence of joint conditions plays a critical role than the boundary conditions.

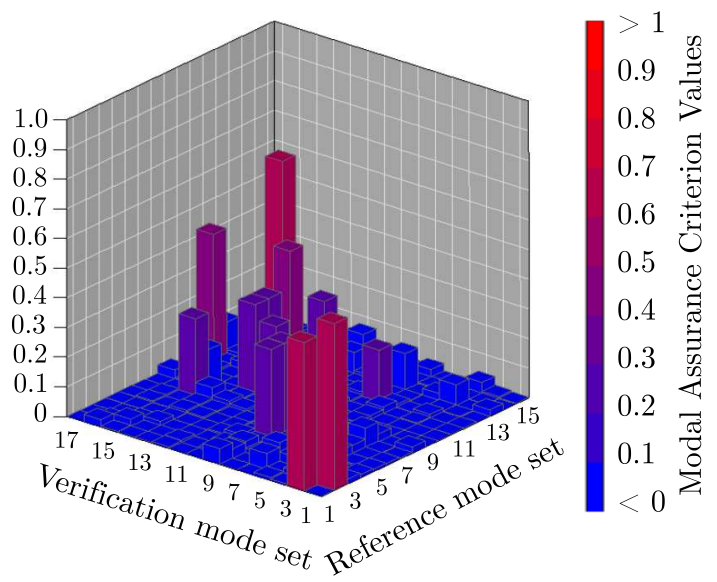

Fig. 11. Modal Assurance Criteria plot for Duct 2 with free-free boundary condition

\section{Conclusions}

Experimental modal analysis is performed for three different rectangular ducts to study the effect of joint conditions on dynamic characteristics of the duct. An ideal rectangular duct shape is considered for numerical calculation. Frequency and RFD plots are used to compare predicted and measured natural frequencies. It is observed from all the cases that experimental modal frequencies are in good agreement with numerical natural frequencies with less than the $10 \%$ error. Mode shapes of predicted and measured results are compared in terms of visual inspection, MAC plot and mode pair table. Low MAC values are observed for Duct 1 and Duct 2 when compared to Duct 3. However, the effect of boundary conditions is also studied on Duct 2 considering free and simply supported boundary conditions. So, based on the observation of all the results, it is concluded that joint conditions play a critical role in mode shape behavior and have a less influence on natural frequency estimation. Further studies are required to incorporate joint stiffness effects in analytical and numerical models to predict dynamic characteristics of rectangular ducts.

\section{References}

1. Allemang R.J., 1999, The Modal Assurance Criterion (MAC): Twenty Years of Use and Abuse, \#309, Proceedings Spie, The International Society for Optical Engineering, 1, 397-405

2. Blevins R.D., 1979, Formulas for Natural Frequency and Mode Shape, Kreiger Publ. Comp., New York

3. Brandon J.A., Cowley A., 1983, A weighted least squares method for circle fitting to frequency response data, Journal of Sound and Vibration, 89, 419-424 
4. Chavan P.N., Venkatesham B., 2015, Free vibration analysis of a rectangular duct with different axial boundary conditions, International Journal of Acoustics and Vibration, 20, 1, 10-14

5. Ewins D.J., 2000a, Modal Testing: Theory and Practice, Letchworth: Research Studies Press, Baldock, Hertfordshire, England

6. Ewins D.J., 2000b, Model validation: correlation for updating, Sadhana, 25, 3, 221-234

7. Fu Z.F., He J., 2001, Modal Analysis, Butterworth-Heinemann, Great Britain

8. Ibrahim S.R., 1999, Fundamentals of time domain modal identification, [In:] Modal Analysis and Testing, Montalvão e Silva J.M., Maia N.M.M. (Eds.), Springer Netherlands, 241-250

9. LeE H.P., 1993, Natural frequencies and modes of cylindrical polygonal ducts, Journal of Sound and Vibration, 164, 1, 182-187

10. Linehan D., Napolitano K., 2012, Accelerometer selection methods for modal pre-test analysis, Sound and Vibration Magazine, 46, 2, 5-8

11. Kennedy C.C., Pancu C.D., 1947, Use of vectors in vibration measurement and analysis, Journal of the Aeronautical Sciences, 14, 603-625

12. MAIA N.M.M., 1988, Extraction of valid modal properties from measured data in structural vibrations, Doctoral dissertation, Imperial College London (University of London)

13. Maia N.M.M., Silva J.M.M., 1997, Theoretical and Experimental Modal Analysis, Research Studies Press, Taunton

14. Schwarz B., Richardson M., Avitabile P., 2002, Locating optimal references for modal testing, Proceedings of the 20th International Modal Analysis Conference, Los Angeles

15. Vacher P., Jacquier B., Bucharles A., 2010, Extensions of the MAC criterion to complex modes, Proceedings of the International Conference on Noise and Vibration Engineering, 2713-2726

16. Xiao H., Pan J., Sheng M., 2011, Vibration characteristics of a cylinder with asymmetries, Proceedings of Acoustics, Australia

17. Zaman I., Khalid A., Manshoor B., Araby S., Ghazali M.I., 2013, The effects of bolted joints on dynamic response of structures, IOP Conference Series: Materials Science and Engineering, IOP Publishing 